Research Article

\title{
3-Dimensional Stochastic Seepage Analysis of a Yangtze River Embankment
}

\author{
Yajun Wang \\ School of Maritime and Civil Engineering, Zhejiang Ocean University, Zhoushan 316000, China \\ Correspondence should be addressed to Yajun Wang; aegis68004@163.com
}

Received 20 August 2015; Accepted 26 October 2015

Academic Editor: Miguel E. Vázquez-Méndez

Copyright (C) 2015 Yajun Wang. This is an open access article distributed under the Creative Commons Attribution License, which permits unrestricted use, distribution, and reproduction in any medium, provided the original work is properly cited.

\begin{abstract}
Three-dimensional stochastic simulation was performed to investigate the complexity of the seepage field of an embankment. Threedimensional anisotropic heterogeneous steady state random seepage finite element model was developed. The material input data were derived from a statistical analysis of strata soil characteristics and geological columns. The Kolmogorov-Smirnov test was used to validate the hypothesis that the Gaussian probability distribution is applicable to the random permeability tensors. A stochastic boundary condition, the random variation of upstream and downstream water level, was taken into account in the threedimensional finite element modelling. Furthermore, the functions of sheet-pile breakwater and catchwater were also incorporated as turbulent sources. This case, together with the variability of soil permeability, has been analyzed to investigate their influence on the hydraulic potential distributed and the random evolution of stochastic seepage field. Results from stochastic analyses have also been compared against those of deterministic analyses. The insights gained in this study suggest it is necessary, feasible, and practical to employ stochastic studies in seepage field problems. The method provides a more comprehensive stochastic algorithm than conventional ones to characterize and analyze three-dimensional random seepage field problems.
\end{abstract}

\section{Introduction}

Seepage analysis of earth dams has to take into account a diverse number of uncertain factors that have significant influence over the geoengineering behavior of the dams. The cause of uncertainty in the seepage field arises mainly from two aspects, namely, uncertainty of the material properties and the specification of the initial-boundary conditions. In view of this, seepage fields such as the head, pressure, and velocity, based on the foregoing uncertainties, should be analyzed as a consequence of a stochastic process with relevant probabilistic distributions. It is important when the generalized stochastic seepage algorithm is being applied to study a dam's stability and performance under seepage and to manage its operation that the uncertainties in the parameters should be properly quantified to ensure credibility to the outcome of the numerical simulation. Stochastic hypothesis testing, as a rule, has to be carried out at first for the random parameters that exist in the governing equation or initialboundary conditions. Furthermore, sensitivity analysis of the uncertain parameters over the stochastic seepage field must be undertaken to better understand the range of possible outcomes predicted by the model. Tsao et al. [1], Griffiths and Fenton [2], have addressed this particular aspect of stochastic seepage analysis. In this paper, the randomness of the seepage field of the main embankment of Yangtze River in Southern Jingzhou was studied with the help of a 3-dimensional anisotropic heterogeneous steady state stochastic seepage numerical model.

As a major levee construction of the state, the main embankment of Yangtze River plays a key role for protection of such zones as Songzi city, Jingzhou city, Gongan county, Shishou city, and Huarong county which hold sway as areas of economic and social significance. The main embankment spans two provincial areas of $2564 \mathrm{~km}^{2}$ from Hubei province to Hunan province, covering $1,737,800$ acres of plantations and population of 1.36 million people to be safeguarded by the embankment system.

A system of underground works runs for $192.32 \mathrm{~km}$ from the inner dike position in the enlarged flood-diversionbasin of Yuanshi city to Wumakou valley in Shishou city. 


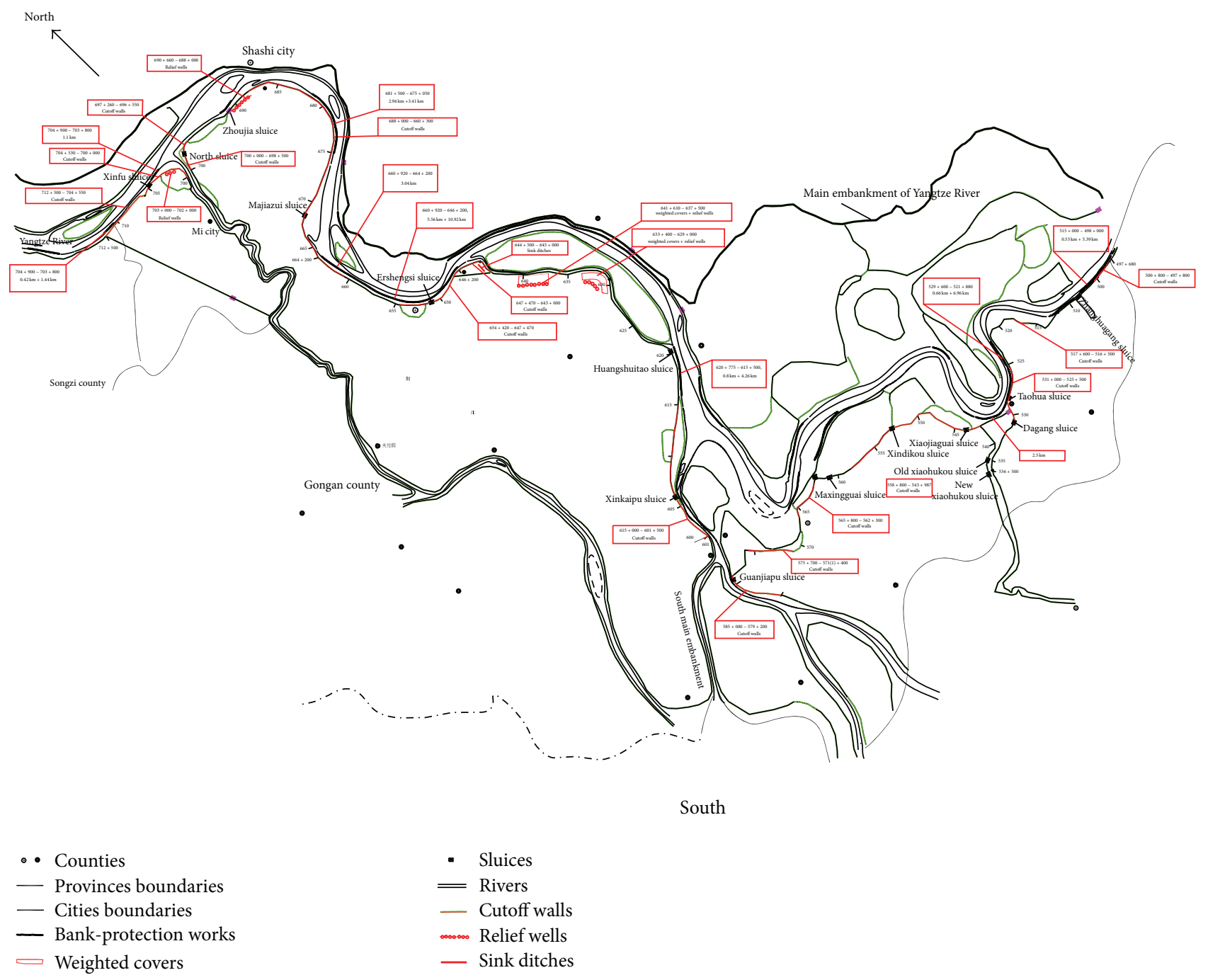

FIGURE 1: Key sites on protection and reinforcement construction of the main embankment of Yangtze River in Southern Jingzhou.

The vital section of $96 \mathrm{~km}$ situated in Gongan city zone, from Beizha area to Ouchi town, is the predominant flood control protection measure for Yangtze River. The local flood-diversion-basin shoulders the safety of Jingzhou city and Gongan county. Furthermore, the total area of flooddiversion-basin in Jingzhou territory covering $921 \mathrm{~km}^{2}$ is used to channel some 5.4 thousand million $\mathrm{m}^{3}$ of flood water (Figure 1).

The complicated embankment system has been eroded by seepage damage in time, some of which may be attributed to poor fills carried out at different times in history. After the flood in 1998, the main body of the dike suffered seepage damage leading to leaks and erosion on the inner slope and toe of the dike. Moreover, these cases of seepage damage remain in the main embankment system, namely, clear water leaks for 12 cases, muddy water leaks for 1 case, formation of sandy channel for 1 case, and dike body cracks for 3 cases, one of which is $100 \mathrm{~m}$ long and the other two are $20 \mathrm{~m}$. Under the circumstances, pertinent research on the seepage characteristics and working behavior of cutoff wall of the main embankment of Yangtze River in Southern Jingzhou territory is extremely important, in particular by using uncertainty theorem including probabilistic numerical method to provide deeper insights.

All of the studies in this paper are established for the working behavior of main embankment of Yangtze River in Southern Jingzhou of Hubei province in China and the corresponding useful data are derived mainly from engineering investigation undertaken by Changjiang Water Resources Commission and Yangtze River Scientific Research Institute.

\section{3-Dimensional Stochastic Seepage Field Model}

The 3-dimensional orthogonal anisotropic heterogeneous steady seepage is governed by the following $[3,4]$ :

$$
\frac{\partial}{\partial x}\left(k_{x} \frac{\partial \mathbf{H}}{\partial x}\right)+\frac{\partial}{\partial y}\left(k_{y} \frac{\partial \mathbf{H}}{\partial y}\right)+\frac{\partial}{\partial z}\left(k_{z} \frac{\partial \mathbf{H}}{\partial z}\right)=0
$$




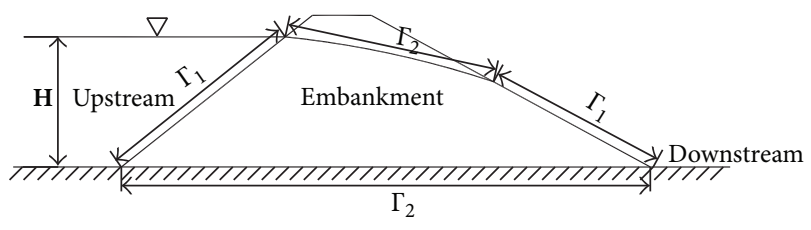

FIGURE 2: Boundary conditions distribution on embankment seepage.

where $x, y$, and $z$ are the principal directions in seepage field; $k_{x}, k_{y}$, and $k_{z}$ given above are designated as orthogonal anisotropic permeability coefficients; $\mathbf{H}$ represents the stochastic head potential distribution array. Two kinds of boundary conditions are generally associated with (1)

$$
\mathbf{H}(x, y, z)_{\Gamma_{1}}=f_{1}(x, y, z) .
$$

Equation (2) is the head boundary condition where $\Gamma_{1}$ represents subgroup on $\mathbf{H}_{\Gamma}$ which is the stochastic boundary head set. $\mathbf{H}_{\Gamma}$ is satisfied by (2) where $f_{1}(x, y, z)$ denotes the head boundary function:

$$
\begin{aligned}
& k_{x} \frac{\partial \mathbf{H}}{\partial x} \cos (n, x)+k_{y} \frac{\partial \mathbf{H}}{\partial y} \cos (n, y) \\
& \quad+k_{z} \frac{\partial \mathbf{H}}{\partial z} \cos (n, z)_{\mid \Gamma_{2}}=f_{2}(x, y, z) .
\end{aligned}
$$

Equation (3) is the flux boundary condition where $\Gamma_{2}$ represents the subgroup on $\mathbf{H}_{\Gamma}$ satisfied by $(3) ; f_{2}(x, y, z)$ denotes the flux boundary function. The normal components of the flux boundary surface are expressed as $\cos (n, x)$, $\cos (n, y)$, and $\cos (n, z)$. Particularly, subgroups $\Gamma_{1}$ and $\Gamma_{2}$ specifying the boundaries in (2) and (3), respectively, are defined under domain space $\boldsymbol{\Omega}_{2}$ which is the stochastic head potential space. Figure 2 shows all the boundary conditions on the seepage field.

The work of Moore [3] and Schevenels et al. [5] has shown that the stochastic seepage field could be expressed as a random process as follows:

$$
\mathbf{K}(\theta) \mathbf{H}(\theta)=\mathbf{F} .
$$

It is here postulated that stochastic permeability matrix $\mathbf{K}(\theta)$ depends linearly on the Gaussian random process $S(X, \theta)$, where $X$ represents the subgroup of parameters containing the random sample subgroup $\theta . F$ is the random turbulence source vector over the seepage field, for example, boundary conditions. Herein, the foregoing numerical model could be formulated as

$$
\left(\mathbf{K}_{0}+\sum_{j=1}^{M} \mathbf{K}\left[\xi_{j}(\theta)\right]_{j}\right) \mathbf{H}(\theta)=\mathbf{F} .
$$

In (5), $\mathbf{K}_{0}$ is the principal systematic permeability matrix and $\mathbf{K}_{j}$ is a matrix function of $\xi_{j}(\theta)$ which denotes the stochastic series $j$ ranging from 1 to $M$ with an independent standard normal distribution. The main cause of seepage field randomness, according to the studies from Dogan and Motz
[6] and Shestakov [7], can be attributed to the stochastic permeability tensor $\mathbf{k}$. Tao and John [8] studied the reliability of the random field including the functions governing microscopic field parameters of the structure material, by which the permeability of the seepage field can be randomly formulated with stochastic medium parameters of diverse probabilistic distributions.

Introducing the interpolation formula $N_{i}(i=$ $1,2, \ldots, m)$, where $i$, ranging from 1 to $m$, refers to the node number of the numerical elements, the conductivity matrix, namely, $\left(\mathbf{K}_{e}\right)_{j}^{P}$, can be deduced as

$$
\left(\mathbf{K}_{e}\right)_{j}^{P}=\iiint_{\tau_{e}} \mathbf{T}^{T}\left(\mathbf{k}_{j}\right) \mathbf{T} \mathrm{d} x \mathrm{~d} y \mathrm{~d} z .
$$

Therefore, the stochastic process $S(X, \theta)$, which simulates the evolution of $\mathbf{K}(\theta)$, could be realized by the stochastic series $\mathbf{k}_{j}$ that, under random steady seepage field condition, relies mainly on permeability perturbation. As for the orthogonal anisotropic seepage model in the integral domain $\tau_{e}$, the permeability tensor under status $j$ is given as

$$
\mathbf{k}_{j}=\left[\begin{array}{ccc}
k_{x} & 0 & 0 \\
0 & k_{y} & 0 \\
0 & 0 & k_{z}
\end{array}\right]_{j}
$$

and the transformation matrix $\mathbf{T}$ as

$$
\mathbf{T}=\left[\begin{array}{cccc}
\frac{\partial N_{1}}{\partial x} & \frac{\partial N_{2}}{\partial x} & \cdots & \frac{\partial N_{m}}{\partial x} \\
\frac{\partial N_{1}}{\partial y} & \frac{\partial N_{2}}{\partial y} & \cdots & \frac{\partial N_{m}}{\partial y} \\
\frac{\partial N_{1}}{\partial z} & \frac{\partial N_{2}}{\partial z} & \cdots & \frac{\partial N_{m}}{\partial z}
\end{array}\right] .
$$

The coordinate transformation is important for dealing with heterogeneous anisotropic seepage field simulation. Due to the rotation of the seepage principal axes, a local to global transformation is to be implemented. To elaborate, if $\alpha_{1}, \alpha_{2}$, and $\alpha_{3}$ and $\beta_{1}, \beta_{2}$, and $\beta_{3}$ are the inclinations and pitch angles of the principal permeability vector (namely, $k_{\xi}, k_{\eta}$, and $k_{\zeta}$ ), then the local coordinate system $(\xi, \eta, \zeta)$ can be transformed to the global system as follows:

$$
\begin{aligned}
& \left\{\begin{array}{l}
x \\
y \\
z
\end{array}\right\} \\
& =\left[\begin{array}{lll}
\cos \beta_{1} \cos \left(\alpha_{x}-\alpha_{1}\right) & \sin \beta_{1} & \cos \beta_{1} \sin \left(\alpha_{x}-\alpha_{1}\right) \\
\cos \beta_{2} \cos \left(\alpha_{x}-\alpha_{2}\right) & \sin \beta_{2} & \cos \beta_{2} \sin \left(\alpha_{x}-\alpha_{2}\right) \\
\cos \beta_{3} \cos \left(\alpha_{x}-\alpha_{3}\right) & \sin \beta_{3} & \cos \beta_{3} \sin \left(\alpha_{x}-\alpha_{3}\right)
\end{array}\right]\left\{\begin{array}{l}
\xi \\
\eta \\
\zeta
\end{array}\right\} \\
& =\mathbf{C} \cdot\{\xi, \eta, \zeta\}^{T},
\end{aligned}
$$

where $\mathbf{C}$ stands for transforming matrix and the following relation is to be set up, too:

$$
\{\xi, \eta, \zeta\}^{T}=\mathbf{C}^{-1}\{x, y, z\}^{T} .
$$




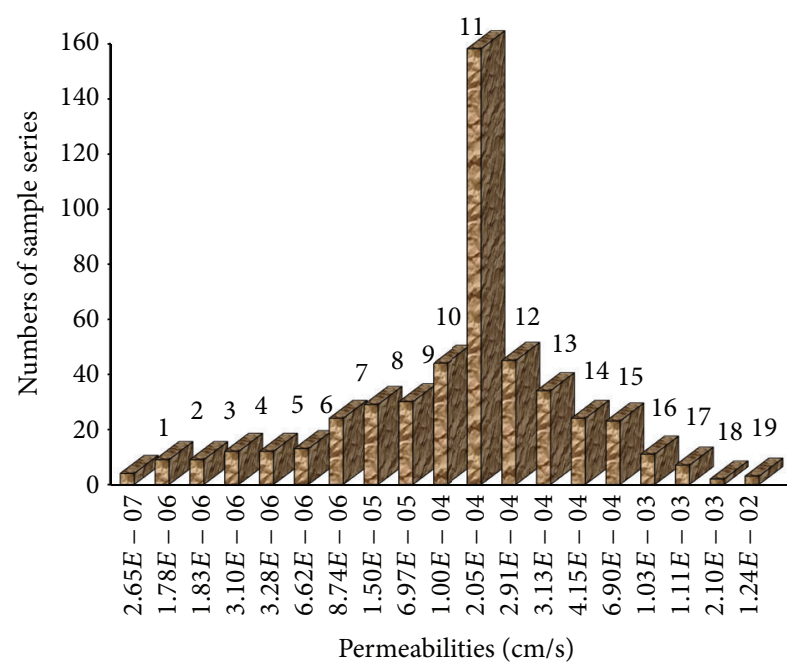

Holocene upper series inclusive of $6,8,10,11,12,13$, and 14 Holocene lower series inclusive of $9,15,16,18$, and 19 Upper Pleistocene series alluvial diluvium inclusive of 7, 17 Lower Pleistocene series alluvial diluvium inclusive of 1, 2, 3, 4, and 5

FIGURE 3: Geologic column on stratum permeability. $\mathbf{H}(\theta)$ :

The interpolating matrix, expressed in (11), is useful for

$$
\mathbf{N}=\left[\begin{array}{llllll}
N_{1} & N_{2} & \cdots & N_{i} & \cdots & N_{m}
\end{array}\right] .
$$

Hence, $\mathbf{H}(\theta)$ can be computed as

$$
\mathbf{H}(\theta)=\sum_{e} \sum_{j=1}^{M} \mathbf{N h}_{e_{j}},
$$

$$
\mathbf{f}_{1}=\left\{f_{1}\left(x_{1}, y_{1}, z_{1}\right) f_{1}\left(x_{2}, y_{2}, z_{2}\right) \cdots f_{1}\left(x_{i}, y_{i}, z_{i}\right) \cdots f_{1}\left(x_{w}, y_{w}, z_{w}\right)\right\}^{T}
$$

where subscript $e$ denotes the accumulation of all the elements and $\mathbf{h}_{e_{j}}$ represents the element stochastic head potential vector expressed as

$$
\mathbf{h}_{e_{j}}=\left\{\begin{array}{llllll}
h_{1}^{j} & h_{2}^{j} & \cdots & h_{i}^{j} & \cdots & h_{m}^{j}
\end{array}\right\}^{T},
$$

where $h_{i}^{j}$ is the nodal stochastic head potential and can be calculated as

$$
h_{i}^{j}=h_{i}(\overline{\mathbf{k}})+\sum_{j=1}^{M}\left(\mathbf{k}_{j}-\overline{\mathbf{k}}\right) \otimes \frac{\partial h_{i}}{\partial \mathbf{k}_{j}}
$$

where $h_{i}$ denotes the expectation of the nodal stochastic head potential. It is computed based on the expectation array $\overline{\mathbf{k}}$ of the stochastic series $\mathbf{k}_{j}$. The operator $\otimes$ indicates that the calculation of (14) is applied just for the orthogonal elements of $\mathbf{k}_{j}$ and $\overline{\mathbf{k}}$.

The random turbulence source vector $\mathbf{F}$ is simulated based on (2) as

$$
\begin{aligned}
\mathbf{F}= & \sum_{j=1}^{M}\left[\mathbf{f}_{1}+\frac{\partial \mathbf{f}_{1}}{\partial x} \Delta x\left(\xi_{j}(\theta)\right)+\frac{\partial \mathbf{f}_{1}}{\partial y} \Delta y\left(\xi_{j}(\theta)\right)\right. \\
& \left.+\frac{\partial \mathbf{f}_{1}}{\partial z} \Delta z\left(\xi_{j}(\theta)\right)\right]_{\mid \Gamma_{1}},
\end{aligned}
$$

where $\mathbf{f}_{1}$ represents the stochastic seepage field freedoms' expectation vector on $\Gamma_{1}$; that is, where $w$ is the half bandwidth of the numerical mesh; $\Delta x\left(\xi_{j}(\theta)\right), \Delta y\left(\xi_{j}(\theta)\right)$, and $\Delta z\left(\xi_{j}(\theta)\right)$ are the random turbulent components on stochastic series $\xi_{j}(\theta)$.

\section{Randomness Expression and Testing on Seepage Field}

3.1. Parameters Formulation on Soil Medium. The main embankment of Yangtze River in Southern Jingzhou is situated on lacustrine facies alluvial deposit, mainly of the Holocene Series of the Quaternary Period. Random Kolmogorov-Smirnov testing was applied in this paper for the permeability of all geological horizons. It can be demonstrated that, under such significant level as $\alpha=1 \%$, the permeability tensor $\mathbf{k}$ submits to Gaussian distribution. Shown in Figure 2 is permeability distribution of the borehole samples which are sufficient in number to cover most typical geological domains in Southern Jingzhou. It also appears in Figure 3 that the local stratum permeability tensor is normally distributed.

In this paper, strata statistical parameters were obtained from geological survey data of sections $580+000$ and $640+000$ of the main dike system (refer to Figures 4 and 5, Table 1). The soil strata, revealed by borehole exploration, consist of fine silt sand, sandy loam soil, silty loam, silty slime and sludge, and so forth and the bedrock. Herein, the local site strata can be classified into two main categories, namely, the relatively pervious sandy strata inclusive of fine silt sand layer and sandy loam soil layer and the relatively impervious clay strata inclusive of silty loam layer, silty slime layer, clay soil, and sludge layer.

For the sake of computational simplicity, the engineering geology of the dike foundation is generalized into three main categories, namely, single structural stratum, doublelayer strata, and multilayer strata. Meanwhile, it is important to note that most of dike foundation sits upon the highly 
TABLE 1: Distribution of material properties for section of $580+500$ and $640+000$.

\begin{tabular}{|c|c|c|c|c|c|}
\hline \multirow{3}{*}{ Category code } & \multirow{3}{*}{ Soil terminology } & \multicolumn{4}{|c|}{ Permeability $/\left(\mathrm{cm} \cdot \mathrm{s}^{-1}\right)$} \\
\hline & & \multicolumn{2}{|c|}{$580+500$ section } & \multicolumn{2}{|c|}{$640+000$ section } \\
\hline & & Expectation $/\left(\mathrm{cm} \cdot \mathrm{s}^{-1}\right)$ & Variation & Expectation $/\left(\mathrm{cm} \cdot \mathrm{s}^{-1}\right)$ & Variation \\
\hline $\mathrm{k} 1$ & Fill mix & $5.0 \times 10^{-5}$ & 0.20 & $3.7 \times 10^{-5}$ & 0.20 \\
\hline $\mathrm{k} 2$ & Loamy soil & (silty) $5.0 \times 10^{-5}$ & 0.10 & $1.0 \times 10^{-5}$ & 0.14 \\
\hline $\mathrm{k} 3$ & Fine silt sand & $5.0 \times 10^{-3}$ & 0.30 & $1.0 \times 10^{-3}$ & 0.30 \\
\hline $\mathrm{k} 4$ & Silty slime & $1.0 \times 10^{-6}$ & 0.27 & $1.0 \times 10^{-5}$ & 0.22 \\
\hline $\mathrm{k} 5$ & Sandy loam soil & & & $8.8 \times 10^{-4}$ & 0.10 \\
\hline $\mathrm{k} 6$ & Midcoarse sand & & & $1.0 \times 10^{-2}$ & 0.20 \\
\hline
\end{tabular}

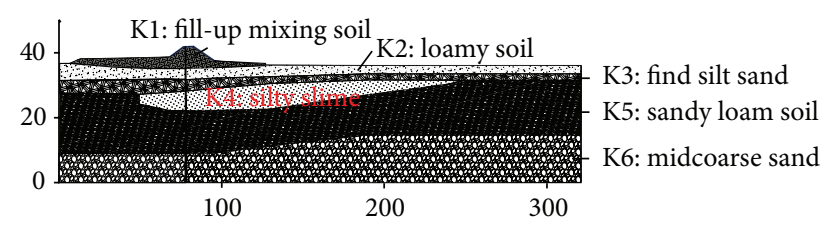

FIGURE 4: Material property on $640+000$ section.

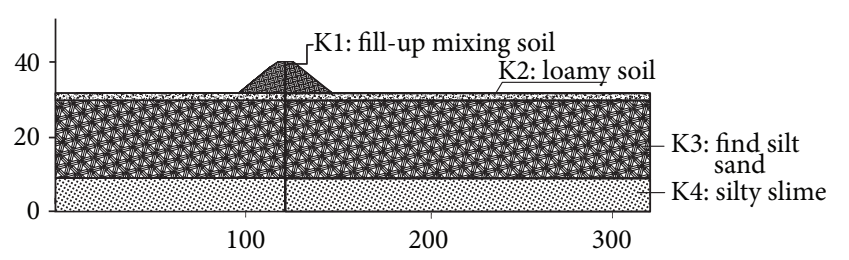

Figure 5: Material property on $580+500$ section.

pervious sand stratum. Seepage field characteristics on these strata, based on the studies from Fenton and Griffiths [9], could be described as the following: due to a great difference of hydraulic conductivities of the diverse strata, such vectors distributions as head and flux and so forth are earmarked by significant variation. River water corroding and forming hydraulic channel through the dike foundation may run towards the dike backside civil block when the river is rising during the flood period above the dike backside height; sandy foundation may become soaked; soil block-floating and piping under seepage pressure may result, by which seepage damage and failure in dike foundation are so severe that the whole dike section may be breached.

3.2. Randomness on Permeability. With the hypothesis that permeability tensor submits to Gaussian distribution and flux submits to logarithmic normal distribution, Tsao et al. [1] realized from his studies using partial-mean stochastic finite element numerical simulation on the basis of random field that only when the boundary condition is very simple could the simulation be implemented properly or else the flux stochastic output is apt to fluctuate unstably with permeability turbulence. In this paper, permeability tensor under hypothesis testing submits to Gaussian distribution over independent stochastic seepage field; namely, $\mathbf{k} \in \boldsymbol{\Omega}_{1}\left(\boldsymbol{\Omega}_{1}\right.$ is random vector space $)$, with which Monte
Carlo stochastic simulation was applied successfully. This conclusion also concurs with the former studies in Figure 3. Particularly, independent double random numbers, namely, $r_{1}$ and $r_{2}$, which submit to rectangular distribution on interval $[0,1]$, could be specified by following (17) where $y_{1}$ and $y_{2}$ stand for two independent random numbers submitting to standard normal distribution. Parameter is determined by computation accuracy:

$$
\begin{aligned}
& r_{1}=\exp \left[-\frac{1}{2}\left(y_{1}^{2}+y_{2}^{2}\right)\right], \\
& r_{2}=\frac{1}{2 \pi}\left[\arctan \left(\frac{y_{2}}{y_{1}}\right)+c\right] .
\end{aligned}
$$

3.3. Randomness on Boundary Condition of Seepage Field. Flood flux in the valley catchment area of Southern Jingzhou, mainly from the upstream of Yangtze River, is characterized with continual high water level and great peak flow. By the research of Mansur et al. [10], the dike system process is very sensitive to outer water turbulence, with which the studies on seepage field head boundary condition randomness are of importance. According to statistical analysis, the studies show that this statistical hypothesis accords closely with monitoring outcomes on site that the downstream-upstream water level in Southern Jingzhou valley catchment area of Yangtze River submits to probabilistic normal distribution. Consequently, the local downstream-upstream water level data in this paper are considered to be independent stochastic variables submitting to normal distribution; namely, $\mathbf{H}_{\Gamma} \in \boldsymbol{\Omega}_{2}$ $\left(\boldsymbol{\Omega}_{2}\right.$, as the stochastic head potential space, has variation of $10^{-5} \sim 10^{-4}$ ).

3.4. Research and Comparison on Randomness of General Seepage Field. On the basis of the foregoing studies, a 3-dimensional anisotropic heterogeneous steady stochastic seepage analysis program is developed here and applied on typical dike systematic sections $580+000$ and $640+000$ where comparisons are made of deterministic and stochastic seepage vectors.

The characteristics of anisotropy and heterogeneousness of seepage field have to be considered when the numerical model grid is being developed (Figure 6). Herein, the same material block should be covered under the corresponding element category in order to induce the rule of randomness on the field medium. Numerical simulations include preand postinstallation of cutoff wall of the main dike sections 


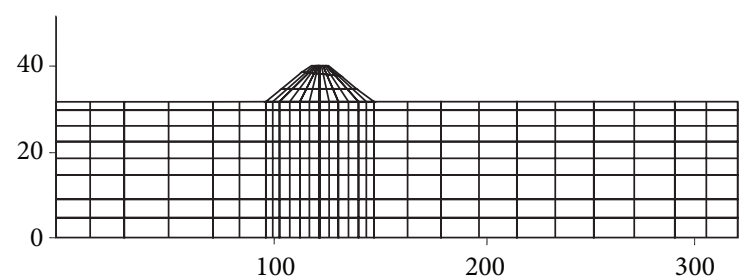

(a) $580+500$ dike section

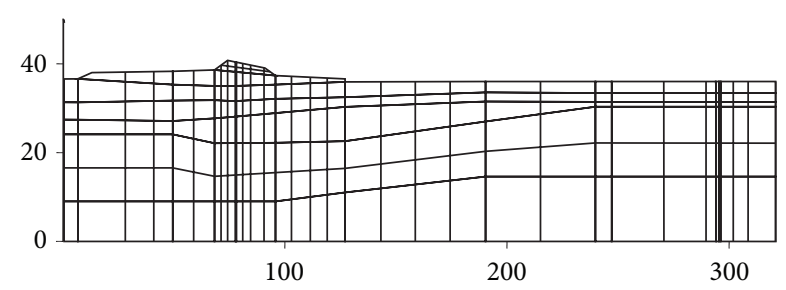

(b) $644+000$ dike section

Figure 6: Grid mesh schemes (unit: m).

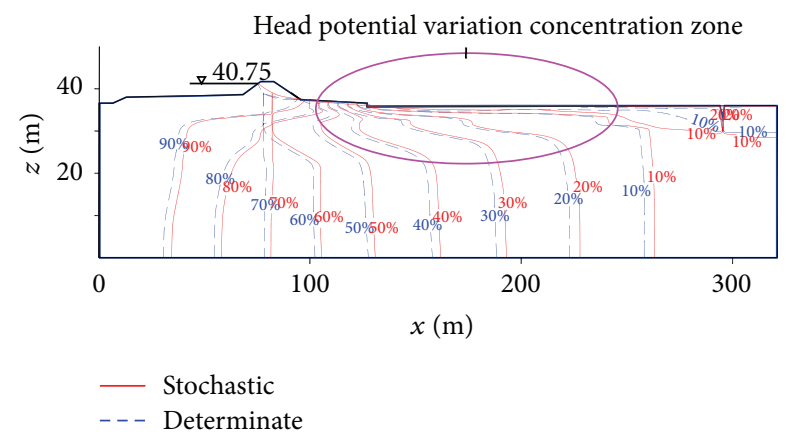

FIGURE 7: Comparison of potential head variation, respectively, from determinate and random seepage field analysis for $644+000$ section after setup seepage cutoff wall.

under deterministic and stochastic processes. Particularly, the behavior of the dike under the downstream relief ditch is calculated for section $640+000$ using the model grid shown in Figure 6. The corresponding determinate and stochastic seepage field distributions are computed and shown in Figure 7 (owing to vaster span of the embankment section than the strata depth, distances in the $z$-direction and $x$ direction have been scaled $2: 1$ for all the head potential contour figures).

With reference to Figure 7, it is evident that there is a difference in the head potential distributions of determinate and stochastic seepage fields under complex boundary conditions. Figure 7 shows the head potential distribution of the diverse strata under the determinate behavior to be homogeneous, with which it is acknowledged that, without variation in the medium parameters, the intrinsic turbulence of head potential distribution due to geological randomness fails to be expressed properly. On the contrary, the stochastic behavior from Figure 7, namely, random seepage field distribution, shows clear concentration of head potential particularly in upper semipermeable layer and sink ditch area, while being relatively uniform in the lower less permeable layer. It can

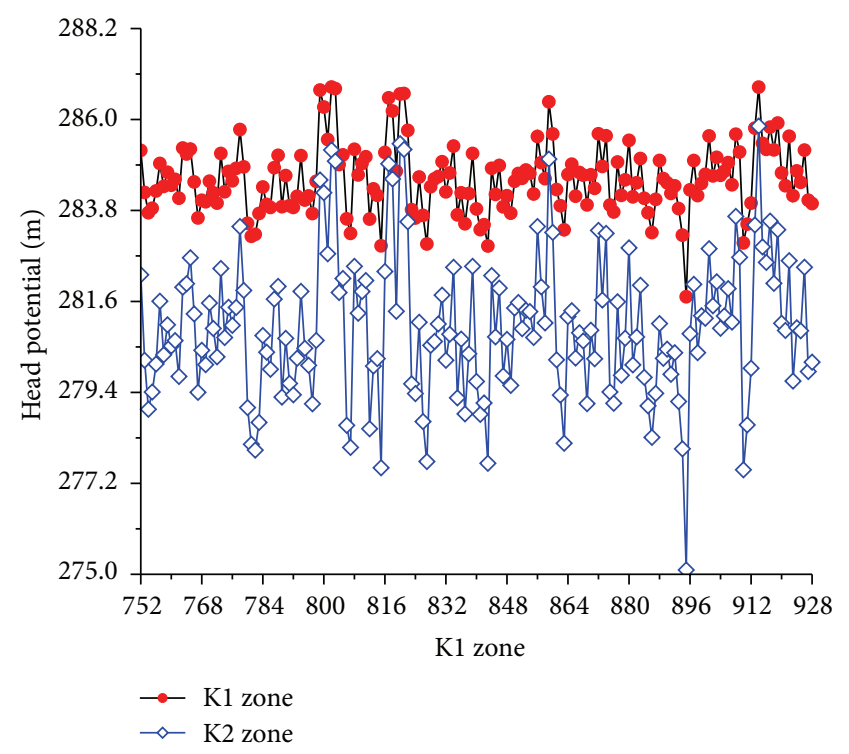

FIGURE 8: Head distribution comparison on $580+500$ section stratum zone (simulation for 762-1000 times).

be surmised that the random head potential distribution of stochastic seepage field with parameter fluctuation could highlight dodging-flow against less permeable strata layer and inclining-flow towards more permeable ones (from Figure 7). According to this figure, the stochastic head potentials concentrate in the upper highly permeable stratum more intensely than determinate ones; the lower less permeable layer of the lenticle is contained in this zone where the stochastic head potentials are 10 percent higher than the determinate ones and the monitoring information on site approaches the random numerical simulation data.

Figure 8 reveals a further tendency of random seepage field that diverse strata under great stochastic variation produce greater consistency. As a conservative hypothesis coupled with the studies from Anisimov [11], it is assumed that the vector field $\underline{\mathbf{H}}:(\theta), \theta=\theta(\underline{\mathbf{k}})$, submits to probabilistic normal distribution; namely, $\underline{\mathbf{H}} \sim N\left(\mu, \sigma^{2}\right)$. The corresponding probabilistic hypothesis testing is carried out in this paper. Pearson $\chi^{2}$ testing is first applied under confidence $1-\alpha=1-0.05=0.95$ to analyze the head potentials distribution of main dike system. Here, two systems, that is, observation frequencies $\left\{m_{1}, m_{2}, \ldots, m_{k}\right\}$ on head and theoretical frequencies $\left\{e_{1}, e_{2}, \ldots, e_{k}\right\}$ on head, are incorporated in the statistical equation as

$$
\chi^{2}=\sum_{i=1}^{k} \frac{\left(m_{i}-e_{i}\right)^{2}}{e_{i}}
$$

and the foregoing hypothesis can be proved when statistical functional value of (13) submits progressively to $\chi^{2}$ distribution with freedom of $f=k-1$, where $k$ is the frequency superior limit. Furthermore, this process can be expressed 


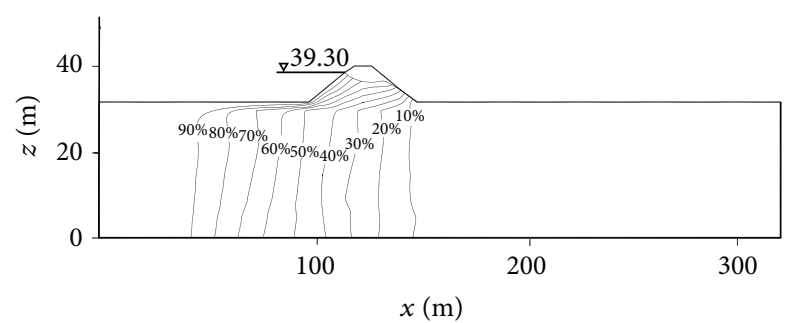

FIGURE 9: Distribution of random seepage field for $580+500$ section before setup seepage cutoff wall.

practically as that foregoing hypothesis can be accepted under confidence $1-\alpha$ when the relation below holds:

$$
\sum_{i=1}^{k} \frac{\left(m_{i}-e_{i}\right)^{2}}{e_{i}} \leq c_{1-a, f},
$$

where $c_{1-a, f}$ represents fractile with cumulative probabilistic value of $(1-\alpha)$ under $\chi^{2}$ distribution.

In this paper, the relative statistical process is applied as $\chi^{2}=\sum_{i=1}^{k}\left(m_{i}-e_{i}\right)^{2} / e_{i}=0.753208$ and owing to $c_{1-a, f}=$ $c_{0.95,3}=7.81$, and it is conspicuous that $\chi^{2}<c_{0.95,3}$. Thereby, this hypothesis could be accepted. Secondly, KolmogorovSmirnov testing is implemented under significant level $\alpha=$ $5 \%$ whether the normal distribution hypothesis is also able to be accepted.

Moreover, a second hypothesis that stochastic head potentials submit to logarithmic normal distribution (namely, $\underline{\mathbf{H}} \sim L N\left(\mu, \sigma^{2}\right)$ ) is tested under confidence $1-\alpha=1-0.05=0.95$ with Pearson $\chi^{2}$ test. However, in view of the differences between observed values and theoretical values, respectively, under normal distribution hypothesis and lognormal distribution giving $\left.0.753208\right|_{N\left(\mu, \sigma^{2}\right)}<\left.2.784043\right|_{L N\left(\mu, \sigma^{2}\right)}$, normal distribution hypothesis of head potential is more applicable.

As a conclusion for these analyses, the head potentials of the stochastic seepage field submit to normal distribution. It also reveals that random simulation is superior to the deterministic one by being able to objectively express higher accumulation of head potential in the material turbulent area up to 2 times than the latter. Moreover, with the shrinkage under stochastic numerical calculation of seepage path by $2 / 3$ of deterministic one, the eventual random failure hydraulic gradient has been escalated 6 times compared with conventional computation.

\section{Stochastic Seepage Field Simulation on Main Embankment of Yangtze River in Southern Jingzhou}

With reference to Figures 9 and 10, the head potential distribution of $580+500$ random seepage field fluctuates quite extensively, especially the head potential magnitude before installation of the cutoff wall. Conversely, the head potential dissipates over most zones after installation of the cutoff wall.

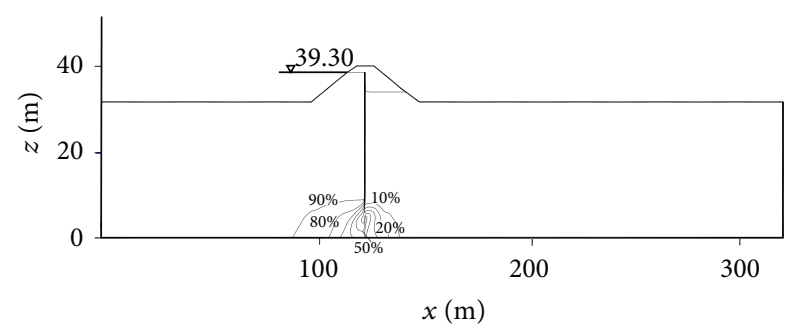

(a)

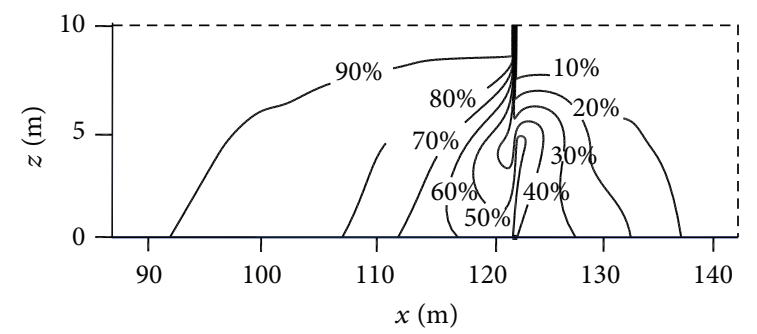

(b)

FIGURE 10: Distribution of random seepage field for $580+500$ section after setup seepage cutoff wall.

These characteristics show that such complex boundary conditions as cutoff walls influence the randomness of seepage field vector distribution significantly.

The main embankment of Yangtze River in Southern Jingzhou, with constitutional strata in most zones [12], is earmarked by great variation of the material parameters. Figure 11 supports the notion that the horizontal effect of an impervious element is as a whole, with intervention of cutoff wall, more effective than the vertical one. Particularly, the head potentials of the deep layer in the vertical direction come into focus when the magnitude of the stratum permeability increases. The subfigures of Figure 11, showing the head distribution of the grid model, indicate the distributing characteristics of the diverse strata's head variation in all direction (head potential without dimension).

Based on the foregoing studies, it is deduced that randomness of seepage field is related not only with the medium parameter variation but also with the macrodistribution of strata and reinforcement structure, for example, cutoff wall whose intervention induces the turbulence of seepage field head distribution variation and leads to the readjustment of stochastic head potentials with the breach against original soil geological layer [13].

With reference to head distribution on $580+500$ section (Figures 12 and 13) under two scenarios, namely, presetup and postsetup of cutoff wall, stochastic simulation results display the concentration profile that led to the shrinkage of the seepage path $20 \mathrm{~m}$ at most. In particular before the setup of cutoff wall, the failure gradient of the random seepage field is increased up to 10 times that under deterministic working behavior. Meanwhile, according to Figure 11, head potential along vertical point group shows high sensitivity to permeability variation with whose value increases from 0.1 to 0.3 , and the head of the stochastic seepage field gains $1.8 \%$. 


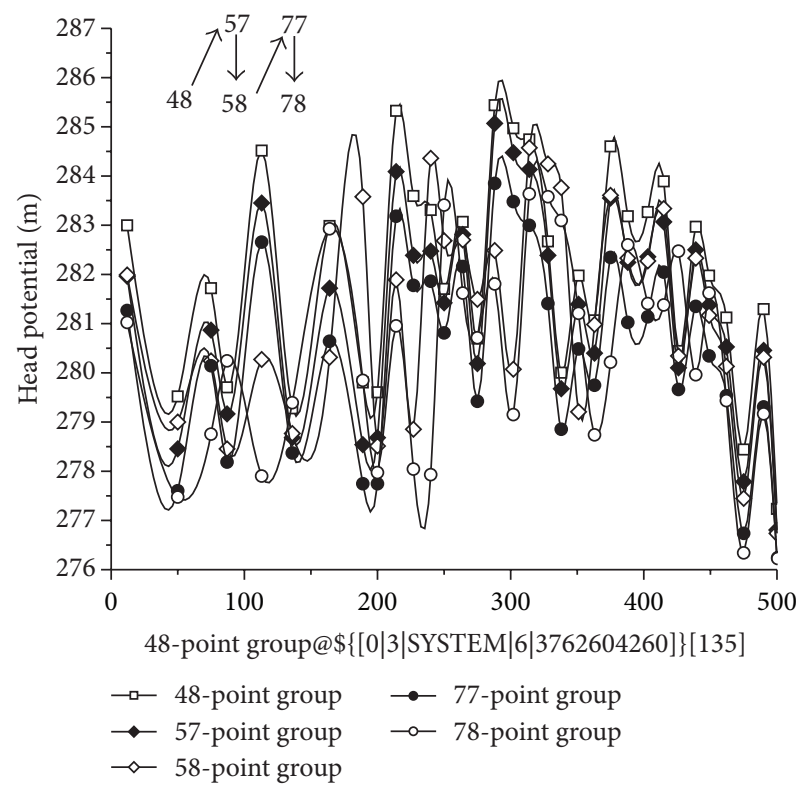

(a) Permeability variation 0.1

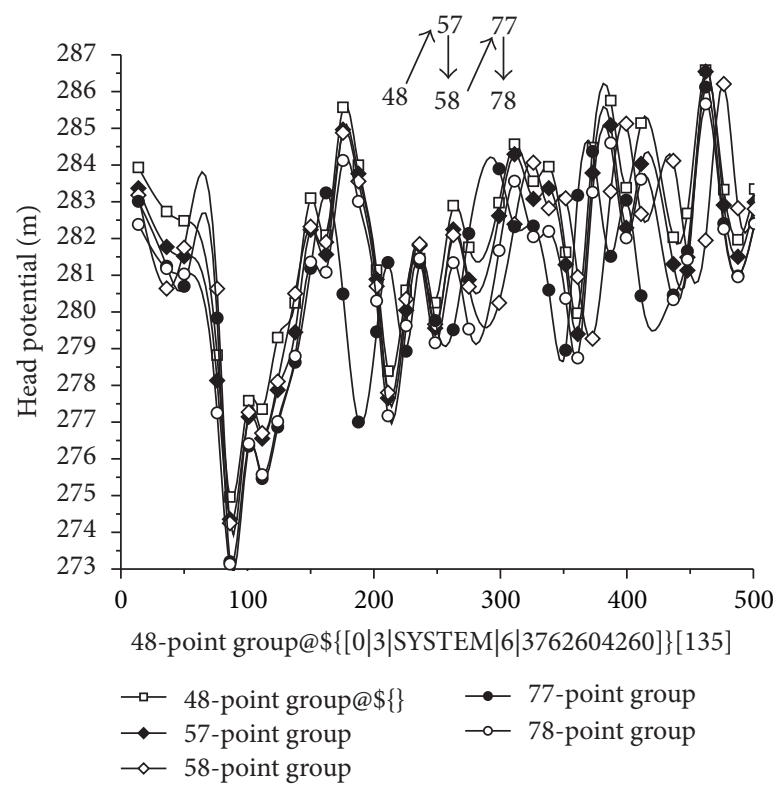

(b) Permeability variation 0.3

FIGURE 11: Distribution characteristics of water head along vertical point group on section $580+500$ for material of k3.

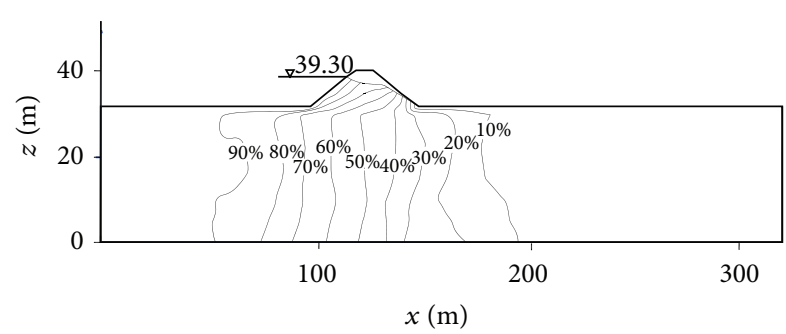

FIGURE 12: Distribution of determinant seepage field for $580+500$ section before setup seepage cutoff wall.

\section{Stochastic Characteristics of Impervious Structure and Boundary Conditions of Seepage Field on Main Embankment of Yangtze River in Southern Jingzhou}

The cutoff walls are installed along the original dike crown line or reinforced one of the main dike systems. The construction process of cutoff wall in addition to the intervention of the sink ditch complicates the randomness of the main dike seepage field even more. Owing to these uncertainties as well as the stochastic fluctuation of boundary head level, it is necessary to rigorously analyze the randomness of complex boundary conditions and their influence over the whole seepage field distribution.

5.1. Randomness on Cutoff Wall. As a rule, the cutoff wall axis should run along the original dike crown line and set back from the dike abutment $1.5 \sim 2.0 \mathrm{~m}$. When the dike bodies have been reinforced or civil architectures are present at concrete pavement outside the dike system, the wall axis can be

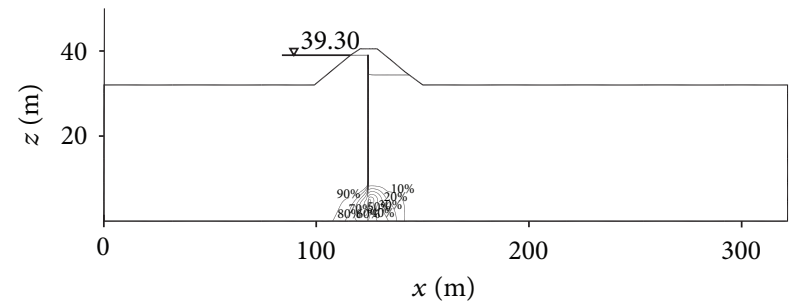

(a)

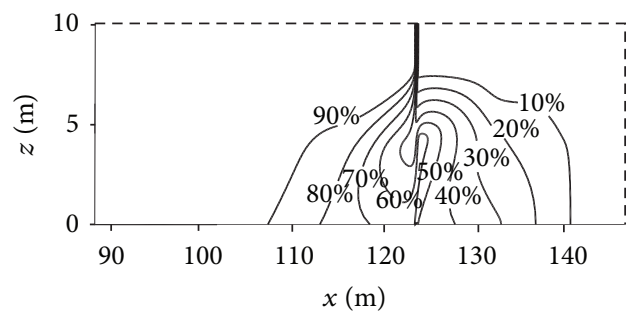

(b)

FIGURE 13: Distribution of determinant seepage field for $580+500$ section after setup seepage cutoff wall.

adjusted according to these engineering criteria: enabling the cutoff wall axis to zigzag along the dike crown line or designing the whole wall axis length $97.484 \mathrm{~km}$. The altitude of cutoff wall is $0.3 \mathrm{~m}$ lower than dike crown altitude. The bottom line of cutoff wall should be embedded into the lower impervious bed layer with sufficient depth: $1.5 \mathrm{~m}$ embedding depth for clay and silty slime and $2.0 \mathrm{~m}$ for loamy soil and silty loam.

The construction sequence for cutoff wall, based on the local technology and construction environment, is as follows: first a deep-agitating concrete wall is formed by plastic 


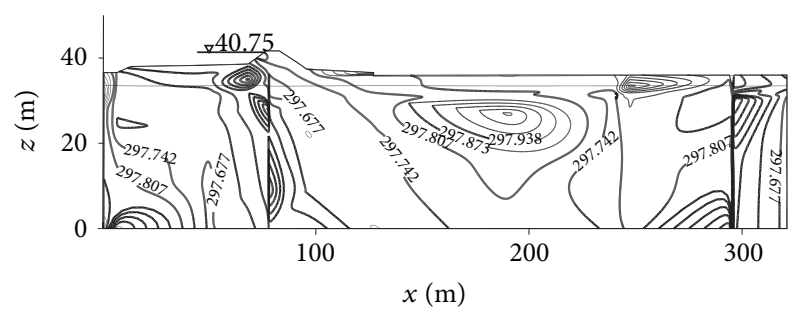

FIGURE 14: Distribution of expected value of random seepage field for $644+000$ section after setup seepage cutoff wall (unit: $\mathrm{m}$ ).

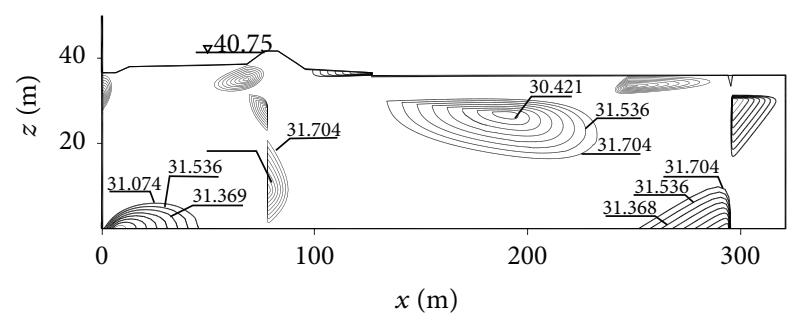

FIGURE 15: Distribution of variance of random seepage field for $644+$ 000 section after setup seepage cutoff wall (unit: $\mathrm{m}^{2}$ ).

wall cutting process, with boring or grooving construction; secondly, due to the obstruction of intervening dikes or civil structures, the wall material in some dike sections can be substituted with high-pressure cement grout layer. The controlling parameters of wall material are presented in Table 2.

The thickness of the cutoff wall can be determined by function $d=H / J$, where $H$ is the maximal working head that is designated to fluctuate as $7 \sim 9 \mathrm{~m}$ according to local terrain and perennial engineering design water level. So, the cutoff wall thickness should be above $150 \mathrm{~mm}$ even beyond $200 \mathrm{~mm}$ to conservatively safeguard the hydraulic reliability. The most noteworthy feature of the stochastic seepage field reinforced with cutoff wall is that head potential drops more clearly through the less pervious stratum [14].

In Figures 14 and 15, randomness of seepage field under complex stochastic boundary conditions is analyzed by calculating the expected head potential. Comparing the foregoing geological section and Figure 14, it can be deduced that the expectation of head potential keeps lower near the lenticle stratum than the sandy layer. Owing to the intervention of rigid wall, local head probabilistic distribution fluctuates turbulently, with head potential maximal and mean square variance values at $31.7 \mathrm{~m}$ and 0.2 , respectively. The intersection zone beneath the dike body absorbs most of the higher expected head potential when without the influence of cutoff wall. On the contrary, when being reinforced by rigid wall, head potentials begin to shift towards the highly pervious stratum and diffuse in sandy layer. Although the distribution of the variance of head potential is unaffected by the intervention of cutoff wall, the head potential variation across the wall is increased.

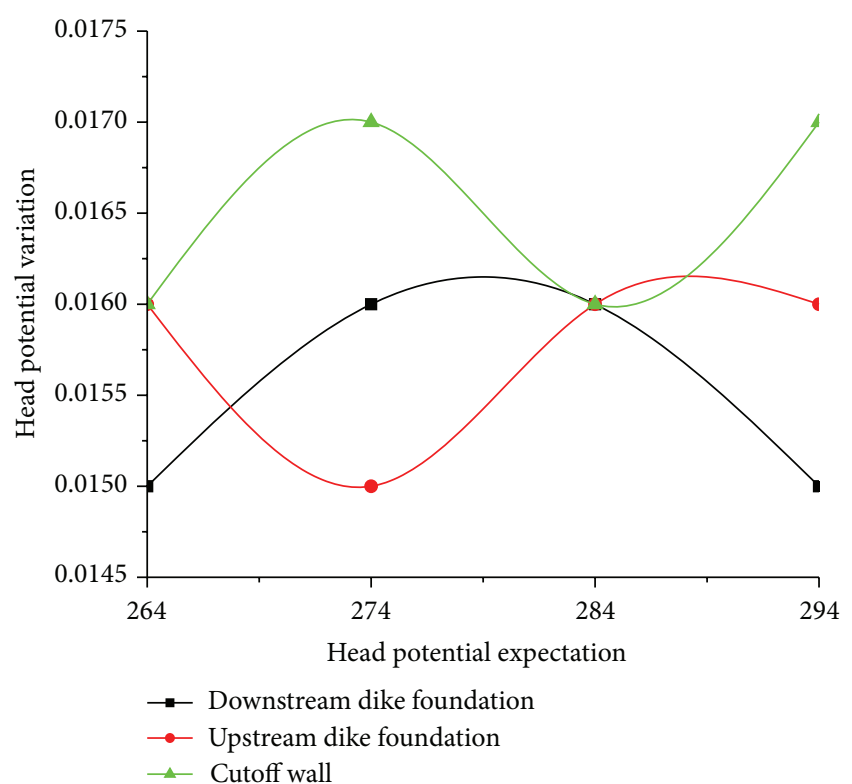

FIGURE 16: Comparison of variation of water head potential in foundation upstream and downstream of dike foundation.

Comparisons of the head potential downstream and upstream of the dike foundation are presented in Figure 16. Also with reference to Figure 16, the contrast of head potential between the dike foundation strata and wall zone shows that the latter is $0.017 \sim 0.018$ higher than the former. Based on the foregoing analysis, there is a greater disturbance in respect of the stochastic seepage field due to the vertical cutoff wall installation [15].

5.2. Randomness on Downstream and Upstream Head Boundary Conditions. From the foregoing studies, the head potential distribution of the main dike seepage field is as a rule inclined to be monotonous with uniform permeability or small variation of upstream and downstream boundary water level. The cause is that the stochastic turbulence of boundary water level decreases and stratum permeability is considered as a deterministic parameter. There is no fluctuating flow in diverse strata when the permeability is deterministic. On the contrary, head distribution begins, with accumulated effect of head boundary condition variation, to concentrate in certain geological zones where the seepage energy attenuates significantly. Seepage deformation due to hydraulic connection flow beneath the dike body intersection may happen. Consequently, impervious barrier should be applied to counter the highly permeable zone. The scale of head variation in upstream dike-ankle is much lower than downstream dike-toe where, under head boundary stochastic turbulence or field permeability random fluctuation, seepage flux exits with head variation increasing (refer to Figure 17). Particularly, the relative height of exit point fluctuates with the increasing of head boundary condition variation, namely, the height of increases with outer water level variation. Furthermore, all these characteristics cause the probability of seepage deformation to increase (refer to Figure 18). 
TABLE 2: Material control parameters of seepage cutoff wall.

\begin{tabular}{lccccc}
\hline Cutoff wall material & Construction process & Thickness of wall/mm & Permeability $/\left(\mathrm{cm} \cdot \mathrm{s}^{-1}\right)$ & $\begin{array}{c}\text { Uniaxial compression } \\
\text { strength/MPa }\end{array}$ & $\begin{array}{c}\text { Permission seepage } \\
\text { gradient }\end{array}$ \\
\hline Clay-cement & Deep agitation & $200 \sim 300$ & $<5 \times 10^{-7}$ & $>1.0$ & 60 \\
Plastic concrete & Grooving & 250 & $<5 \times 10^{-7}$ & $>2.0$ & 60 \\
Cement paste & High pressure injection & 200 & $<1 \times 10^{-6}$ & $>1.0$ & 60 \\
\hline
\end{tabular}

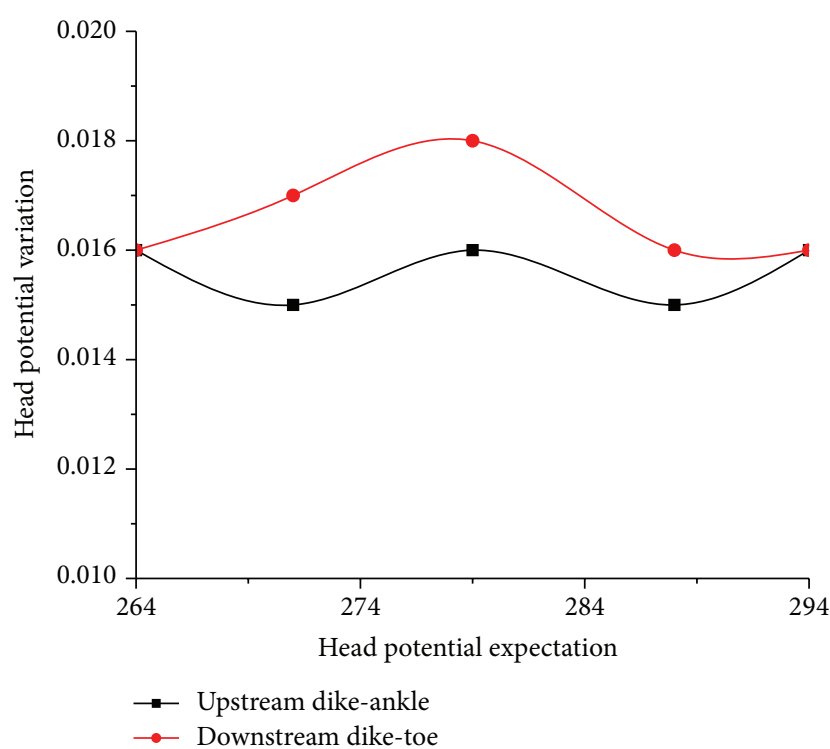

FIGURE 17: Comparison of variation of water head potential on slopes between upstream dike-ankle and downstream dike-toe.

In Figure 18, the overwhelmingly influential effect of head boundary variation is manifested in terms of downstream exit height that determines directly the deformation situation of the seeping exit section. With variation of $13 \times 10^{-5}$, the relative exit height stands at just $0.05 \mathrm{~m}$. When variation of head boundary increases to $13 \times 10^{-4}$, however, the relative exit height increased significantly to $1.4 \mathrm{~m}$, which is serious enough to cause layer-drift over large zone of the exit section.

5.3. Randomness on Relief Well (Leading Sink Ditch). The total length of relief well axis (namely, the axis of leading sink ditch which is designed to cover the gutter and hold 5 $\times 10^{-2} \mathrm{~m}^{3} / \mathrm{s}$ total flux) is $1,020 \mathrm{~m}$ running parallel with the dike axis and $200 \mathrm{~m}$ distant from the dike-toe. The excavation depth as a rule should reach the sandy pervious stratum of dike foundation, for example, the range of $3 \sim 4 \mathrm{~m}$. Figure 19 shows the general structure of sink ditch.

The effect of sink ditch randomness can be observed in Figure 20. When compared with the dike foundation, the expectation value of head potential in the leading sink ditch is lower with the highest difference of about $2 \mathrm{~m}$. On the contrary, the mean square variance of head potentials in leading sink ditch is higher than dike foundation zone with

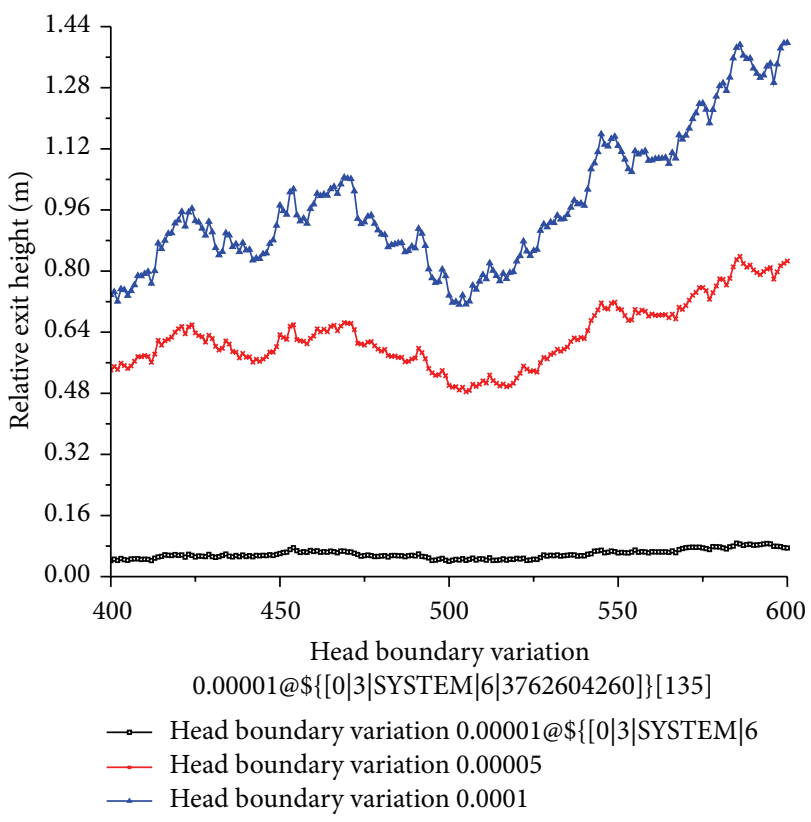

FIGURE 18: Downstream exit height sensitivity to head boundary variation (simulation for 400 600 times without installation of cutoff wall).

the largest difference of $2 \mathrm{~m}$ which reflects that the variation of the former is 4 times the latter at most.

In the next step, hydraulic gradient of the stochastic seepage field of main dike is studied under the influence of relief well and cutoff wall, and it is deduced that variation of seepage field hydraulic gradient increases with the variation of head boundary, whereas the expected value of seepage field hydraulic gradient is observed to be decreasing (refer to Figures 21 and 22), which is in agreement with the research outcome of Mansur et al. [10]. Moreover, it is important that this engineering phenomenon has close relation with such drainage facilities as the relief well set near the tail water. Further study on the complex boundary condition of random seepage field discloses that the installation of relief well on the site where hydraulic cleavage crack often occurs is useful for decreasing the tail water level. Meanwhile, the tail water level variation, even with drainage elements, can disturb the distribution of hydraulic gradient of the exit section. It has been reported that such complex boundary condition as a leading sink ditch is very sensitive to stochastic turbulence of seepage field and the hydraulic gradient is also sensitive to cutoff wall as well as relief well when variation of boundary 


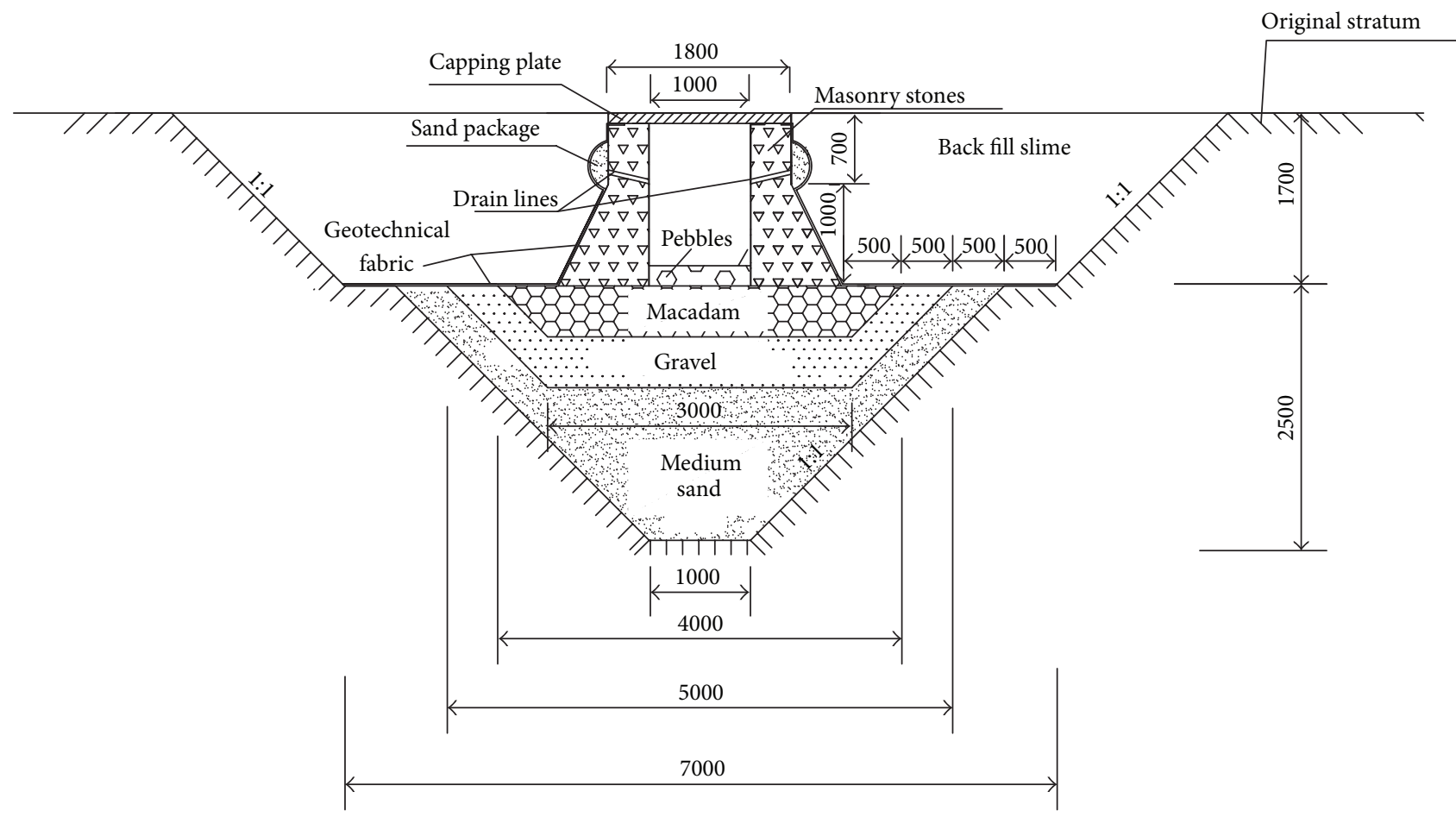

Figure 19: Structure of leading sink ditch (scale/cm).

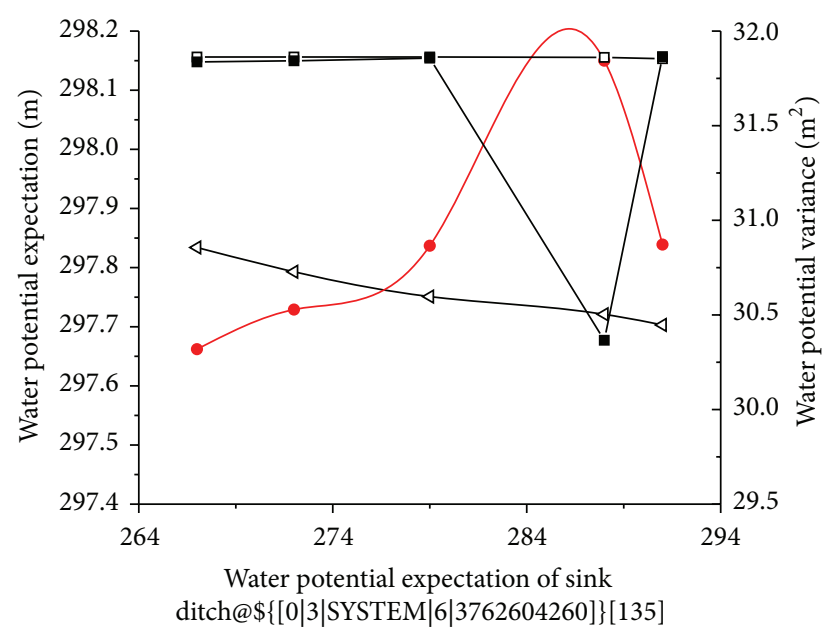

FIGURE 20: Comparison of water head variation on $644+000$ section and leading sink between upstream and downstream foundations.

water level is increasing [16]. Furthermore, the installation of cutoff wall can effectively attenuate the scale of hydraulic gradient of random seepage field.

Referring to Figure 22, the hydraulic gradient variation of the stochastic seepage field, under the influence of random turbulence of relief well and cutoff wall, gains 2 times that before the cutoff wall setup. Although the expected value of hydraulic gradient decreases as a whole by $20 \%$ with cutoff wall, owing to great turbulence in tail zone, such seeping damage, as scattering leaks, in particular muddy leaks occurs (refer to Figure 23).

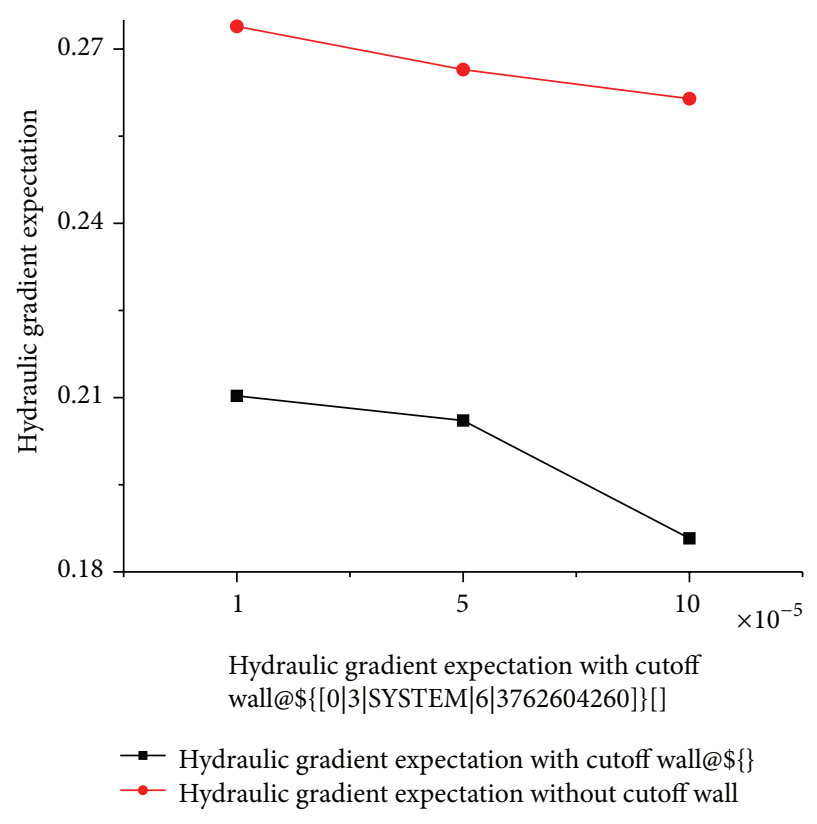

FIGURE 21: Hydraulic gradient expectation sensitivity to head boundary variation.

\section{Conclusion}

By the help of 3-dimensional anisotropic heterogeneous stochastic seepage numerical model program, the randomness of 3-dimensional anisotropic heterogeneous steady stochastic seepage field of the main embankment of Yangtze 


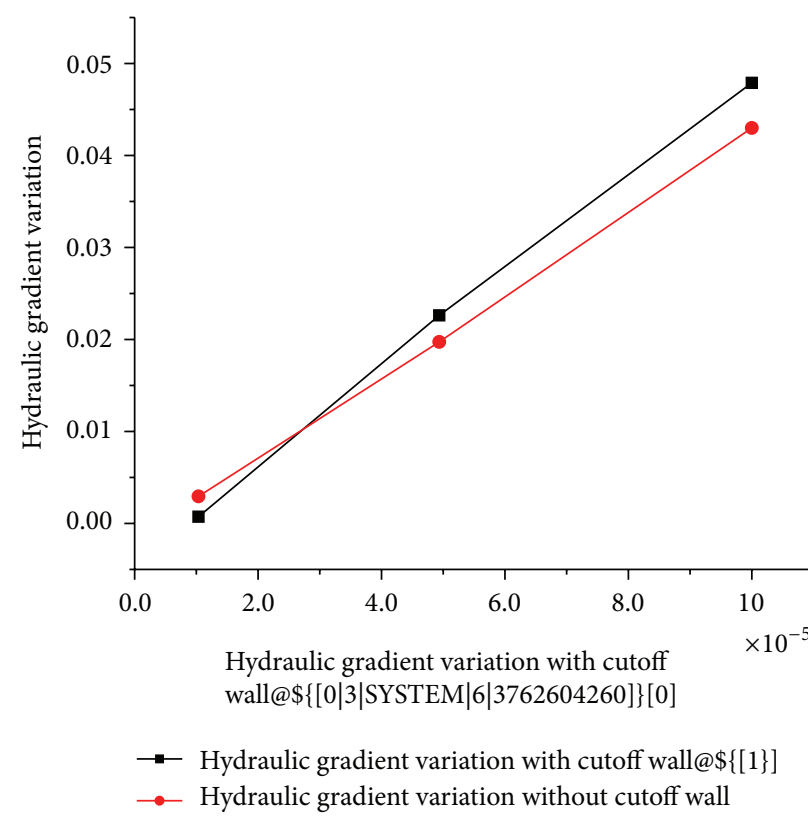

FIGURE 22: Hydraulic gradient variation sensitivity to head boundary variation.

River in Southern Jingzhou is studied comprehensively. This paper has analyzed the influence of strata parameters' stochastic characteristics and boundary water level variation over random seepage field head distribution as well as the stochastic disturbance of complicated boundary conditions over random seepage field. The main conclusions are as follows:

(1) In this paper, the elements of stochastic permeability matrix are random process submitting to independent normal probabilistic distribution.

(2) Although the geological strata cover complicates the whole seepage field, the stochastic numerical analyses on diverse layers show a rule of consistency which verifies the practicability and rationality of algorithm program.

(3) Under random simulation, the head potential in the material turbulent area is, as a whole, 2 times or so that under deterministic process. Furthermore, with the reduction under stochastic numerical calculation of seepage path by $2 / 3$ of deterministic one, eventual random failure of the hydraulic gradient has been escalated 6 times compared with conventional computation.

(4) Downstream section of the dike system, where seepage flux exits, is the concentration zone of head variation of great magnitude. With the accumulated effects of boundary water level variation, the relative height of exiting point group increases and exiting section is enlarged which causes the probability of seepage damage to increase.

(5) With the range of head boundary variation from $13 \times$ $10^{-5}$ to $13 \times 10^{-4}$, the relative exit height increases

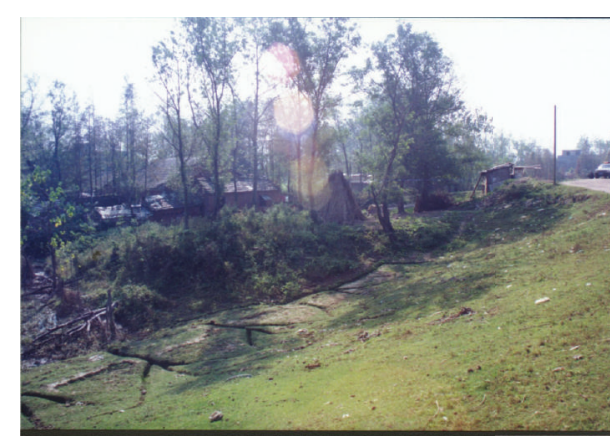

(a) Muddy water leaks

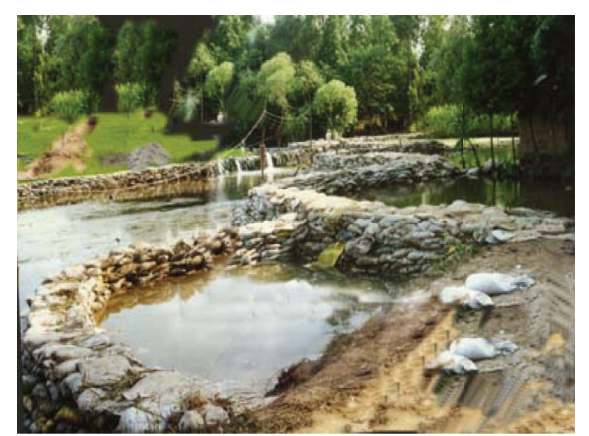

(b) Clear water leaks

FIGURE 23: Scattering leak cases in tail zone of the main embankment of Yangtze River in Southern Jingzhou.

from $0.05 \mathrm{~m}$ to $1.4 \mathrm{~m}$, which increases the tendency to induce layer-drift over large zone of exit section.

(6) Before the installation of cutoff wall, the intersection beneath the dike body is the concentration zone of head of great magnitude; with cutoff wall, concentration of head potential diffuses towards the pervious strata and the head variation of zone near the wall increases by up to $0.017 \sim 0.018$. Consequently, the turbulence influence of the vertical impervious zone against random head potential distribution is shown clearly.

(7) Concentration of stochastic simulation leads to the reduction of seepage path by $20 \mathrm{~m}$ at most. So, particularly before the setup of cutoff wall, the failure gradient of random seepage field is increased up to 10 times that under deterministic conditions. Distribution of head potential along the vertical shows its high sensitivity to permeability variation with the value increasing from 0.1 to 0.3 and the head value of stochastic seepage field gains as a whole $1.8 \%$.

(8) Head variation of the leading sink ditch is 4 times that of dike foundation. It is thus acknowledged that such complex boundary condition as leading sink ditch is very sensitive to the random turbulence of seepage field distribution. The variation of hydraulic gradient of seepage field increases with variation of downstream boundary water level. Meanwhile, the expected values of hydraulic gradient of the seepage 
field decrease with increasing of variation of downstream boundary water level.

(9) Hydraulic gradient variation of stochastic seepage field, under the influential effect of random turbulence of relief well and cutoff wall, gains 2 times that before cutoff wall setup. This will cause muddy leaks that are highly destructive damage in tail zone to occur under many circumstances.

\section{Conflict of Interests}

The author declares that there is no conflict of interests regarding the publication of this paper.

\section{Acknowledgments}

This work was supported by the National Natural Science Foundation of China (Grant no. 51109118), Zhejiang Provincial Natural Science Foundation of China (Grant no. LY14E090001), United Development Project Foundation from Zhejiang Ocean University and Wenzhou University (Grant no. 21188004113), United Development Project Foundation from Zhejiang Ocean University and Hydrochina Huadong Engineering Corporation (Grant no. 21188004013 and 21188006615), and Young Teachers Improvement Project Fund of Zhejiang Ocean University (Grant no. 11042101512).

\section{References}

[1] T. M. Tsao, M. K. Wang, M. C. Chen, Y. Takeuchi, S. Matsuura, and H. Ochiai, "A case study of the pore water pressure fluctuation on the slip surface using horizontal borehole works on drainage well," Engineering Geology, vol. 78, no. 1-2, pp. 105118, 2005.

[2] D. V. Griffiths and G. A. Fenton, "Three-dimensional seepage through spatially random soil," Journal of Geotechnical Engineering, vol. 123, no. 2, pp. 153-160, 1997.

[3] S. M. Moore, "Stochastic fields from stochastic mechanics," Journal of Mathematical Physics, vol. 21, no. 8, pp. 2102-2110, 1980.

[4] S. N. Numerov, "Nonlinear seepage in anisotropic media," in Proceedings of the 15th Congress of the International Association for Hydraulic Research, no. 3, pp. 39-46, Istanbul, Turkey, 1973.

[5] M. Schevenels, G. Lombaert, and G. Degrande, "Application of the stochastic finite element method for gaussian and nongaussian systems," Proceedings of the ISMA, no. 1, pp. 120-124, 2004.

[6] A. Dogan and L. H. Motz, "Saturated-unsaturated 3D groundwater model. I: development," Journal of Hydrologic Engineering, vol. 10, no. 6, pp. 492-504, 2005.

[7] V. M. Shestakov, "Generalized model of plane seepage flow," Fluid Dynamics, vol. 27, no. 1, pp. 141-143, 1992.

[8] H. Tao and W. R. John, "A mathematical model for seepage of deeply buried groundwater under higher pressure and temperature," Journal of Hydrology, vol. 327, no. 1-2, pp. 42-54, 2006.

[9] G. A. Fenton and D. V. Griffiths, "Statistics of flow through a simple bounded stochastic medium," Water Resources Research, vol. 29, no. 6, pp. 1825-1830, 1993.
[10] C. I. Mansur, G. Postol, and J. R. Salley, "Performance of relief well systems along Mississippi river levees," Journal of Geotechnical and Geoenvironmental Engineering, vol. 126, no. 8, pp. 727-738, 2000.

[11] K. I. Anisimov, "Causes of seasonal variations of the seepage regime of earth structures," Hydrotechnical Construction, vol. 22, no. 9, pp. 537-541, 1988.

[12] S. A. Isiorho, F. M. Beeching, P. M. Stewart, and R. L. Whitman, "Seepage measurements from Long Lake, Indiana Dunes National Lakeshore," Environmental Geology, vol. 28, no. 2, pp. 99-105, 1996.

[13] B.-J. Bathe and M. R. Khoshgoftaar, "Finite element free surface seepage analysis without mesh iteration," International Journal for Numerical \& Analytical Methods in Geomechanics, vol. 3, no. 1, pp. 13-22, 1979.

[14] A. M. Antonova and I. I. Moiseikina, "Seepage of a fluid to an imperfect well in a bounded nonhomogeneously anisotropic fissured-porous layer," Journal of Mathematical Sciences, vol. 71, no. 5, pp. 2666-2669, 1994.

[15] F. D. Patrick, A. G. Teamrat, J. C. Paul, J. A. RodríguezPineda, L. Villalba, and R. De la Garza, "Heterogeneous seepage at the Nopal I natural analogue site, Chihuahua, Mexico," Hydrogeology Journal, vol. 20, no. 1, pp. 155-166, 2012.

[16] M. P. Bruen and Y. Z. Osman, "Sensitivity of stream-aquifer seepage to spatial variability of the saturated hydraulic conductivity of the aquifer," Journal of Hydrology, vol. 293, no. 1-4, pp. 289-302, 2004. 


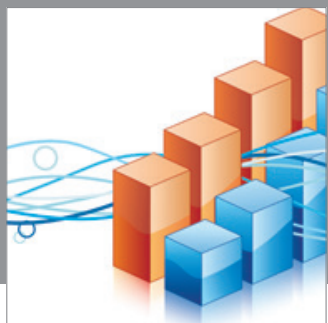

Advances in

Operations Research

mansans

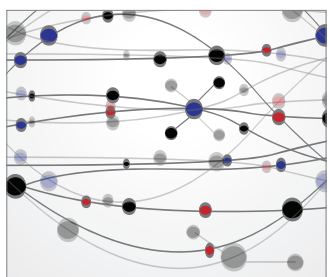

The Scientific World Journal
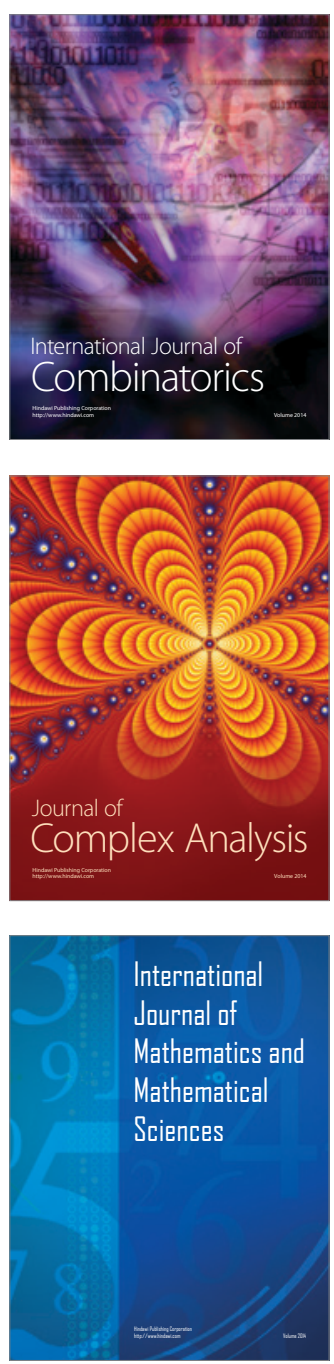
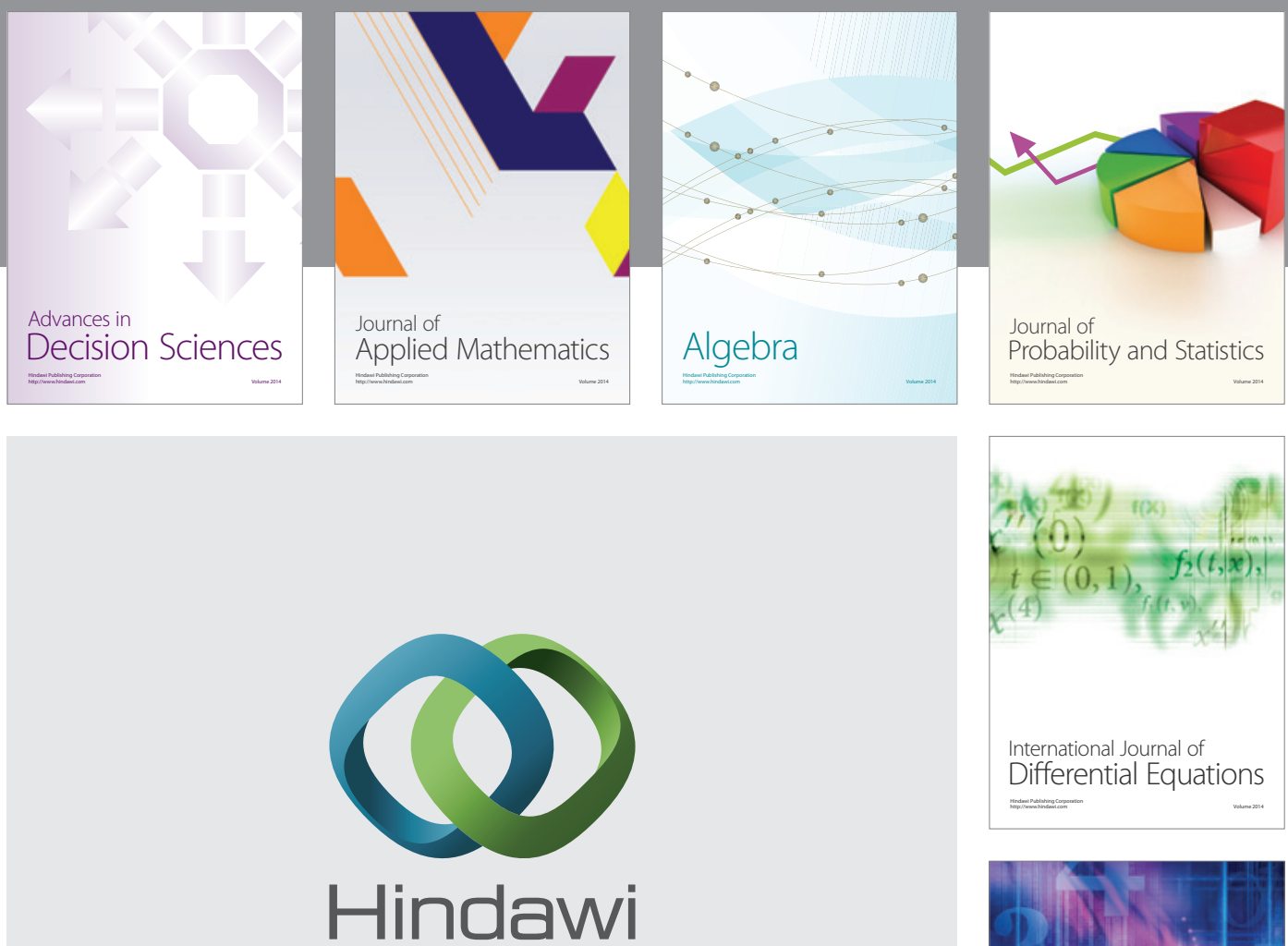

Submit your manuscripts at http://www.hindawi.com
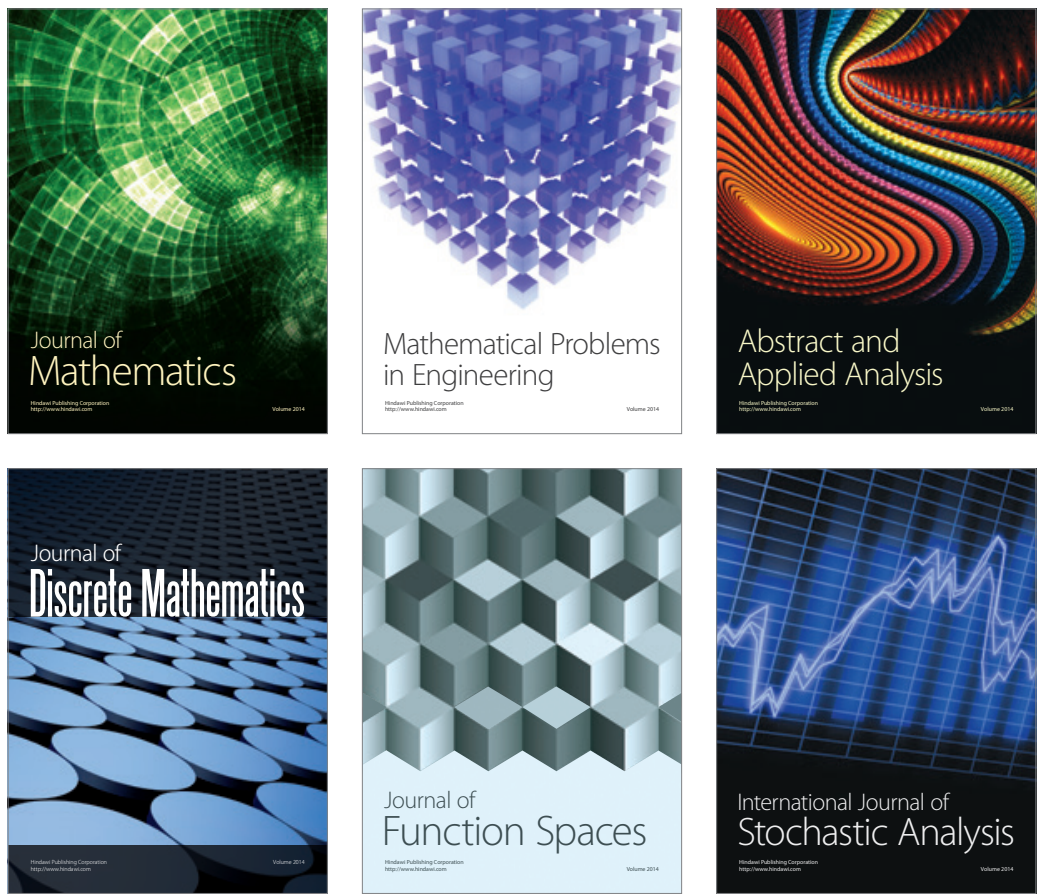

Journal of

Function Spaces

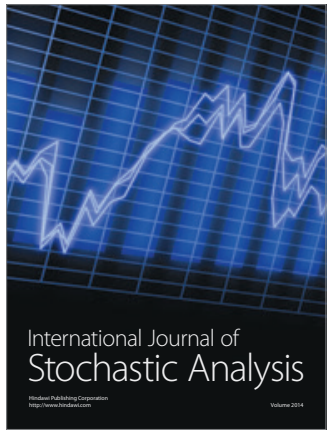

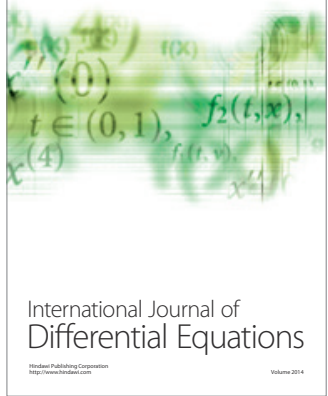
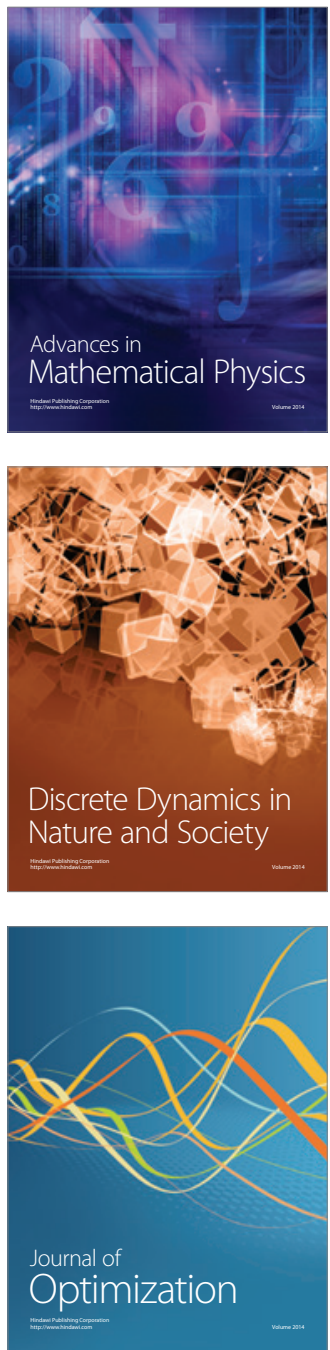